\title{
Time angle ocean acoustic tomography using sensitivity kernels: The forward problem
}

\author{
Florian Aulanier ${ }^{* 1}$, Barbara Nicolas ${ }^{\dagger 1}$, Philippe Roux ${ }^{\ddagger 2}$, and Jérôme I. Mars ${ }^{\S 1}$ \\ ${ }^{1}$ GIPSA-Lab, Université de Grenoble \\ ${ }^{2}$ ISTerre, Université de Grenoble
}

\section{Introduction}

In shallow water oceans, mid-frequency acoustic waves take multiple ray-like paths to go from a source to a receiver. Classically, ocean acoustic tomography (OAT) uses the travel-times (TT) associated to these raypaths to retrieve seawater characteristics (sound speed, temperature...) [1].

In the 80's, geophysicists use the Born and Rytov approximations [2], well known in optics, to introduce the concept of wavepaths instead of raypaths. This allows taking into account the diffraction phenomena occurring at finite frequency to perform tomography $[3,4]$. This concept gives birth the so-called bananadoughnut sensitivity kernels that describe the paradoxical behavior of the seismic wave travel-times [5]. Sensitivity kernels are then brought to ocean acoustics to perform point-to-point sound speed tomography using acoustic wave travel-times [6]. The use of source-receiver arrays and double-beamforming [7, 8] to separate interfering acoustic arrivals coming from the multiple paths, have been used to improve the TT sensitivity kernels method [9]. The use of source and receive arrays also permits to measure the directions-of-arrival (DOA) and the directions-of-departure (DOD) corresponding to each acoustic arrival at the same time as TT. Considering these 3 observables, joint sensitivity kernels of TT, DOA and DOD have been theoretically formulated in view of ocean sound speed inversion [10].

In this article, the forward problem using time-angle sensitivity kernels (T-A-SK) - estimate TT, DOA and DOD variations from known sound speed perturbations - is studied in order to know its strength and limits.

\section{Oceanic context and theoretical background}

Considering a Pekeris waveguide of $1.5 \mathrm{~km}$ in range and $50 \mathrm{~m}$ in depth, and a signal of $2.5 \mathrm{KHz}$ of central frequency and $1.25 \mathrm{KHz}$ of bandwidth, the sound take ray-like paths to travel through the ocean. These paths can be characterized by their observables: travel-times $(\tau)$, directions-of-arrival $\left(\theta_{r}\right)$ and directions-of-departure $\left(\theta_{e}\right)$ as shown on Fig.1.

Using the first order Born approximation [Born et al. 1999], it is possible to link the observable variations $\left(\delta \tau, \delta \theta_{r}\right.$ and $\left.\delta \theta_{e}\right)$ to the sound speed perturbations $(\delta c)$ in a linear way [Aulanier et al. 2011] :

$$
\left(\begin{array}{c}
\delta \tau \\
\delta \Theta_{r} \\
\delta \Theta_{e}
\end{array}\right) \simeq \iiint_{V}\left(\begin{array}{c}
K_{T T}\left(\boldsymbol{r}^{\prime}\right) \\
K_{D O A}\left(\boldsymbol{r}^{\prime}\right) \\
K_{D O D}\left(\boldsymbol{r}^{\prime}\right)
\end{array}\right) \delta c\left(\boldsymbol{r}^{\prime}\right) \mathrm{d} V\left(\boldsymbol{r}^{\prime}\right)
$$

where $K_{T T}, K_{D O A}$ and $K_{D O D}$ are the sensitivity kernels associated to each observable TT, DOA and DOD respectively. The waveguide volume is noted as $V$ and $\mathrm{d} V\left(\boldsymbol{r}^{\prime}\right)$ is an elementary volume located in the waveguide at point $\boldsymbol{r}^{\prime}$.

Therefore, knowing the sound speed perturbation it is possible to estimate the induced observable variation using the T-A-SK.

\footnotetext{
*Florian.Aulanier@gipsa-lab.grenoble-inp.fr

${ }^{\dagger}$ Barbara.Nicolas.Aulanier@gipsa-lab.grenoble-inp.fr

$\ddagger$ Philippe.Roux@obs.ujf-grenoble.fr

§ Jerome.Mars@gipsa-lab.grenoble-inp.fr
} 


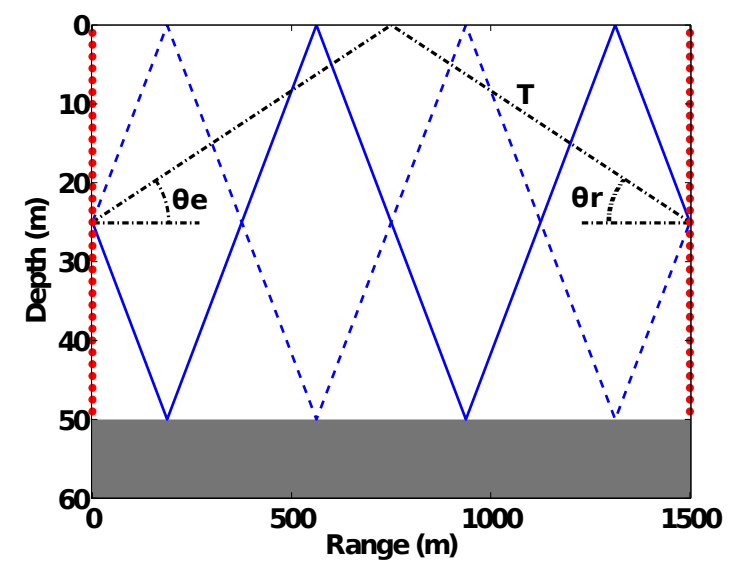

Figure 1: Pekeris waveguide with examples of three possible raypaths. The source and receive array at the beginning and the end of the waveguide have 97 elements evenly spaced by $0.5 \mathrm{~m}$ i.e. . These observables - measured with source-receiver arrays and the double beamforming technique [Roux et al. 2008 ] - vary when the sound speed distribution changes in the waveguide.

\section{The T-A-SK forward problem tested on parabolic equation simulations}

\subsection{Principle of the Test}

In order to analyze, the results of the forward problem using T-A-SK, the T-A-SK estimates of the observable variations are compared to the observable variations measured on parabolic equation $(\mathrm{PE})$ simulations [11]. The waveguide used in simulations is the same as the one described on Fig. 1. A set of 550 rays is selected including rays with 2 to 12 reflections on the surface and the bottom recorded with 25 pairs of source-receiver arrays. We focus on some test cases chosen to analyze the influence of the main parameters involved in tomography configuration, which are:

- the sub-array sizes,

- the sound speed perturbation locations within the waveguide,

- the perturbation values,

- the perturbation areas,

- and the case of multiple perturbations of opposite signs.

\subsection{The Sub-Array Size}

Sub-arrays of $15 \mathrm{~m}, 21 \mathrm{~m}, 31 \mathrm{~m}$ and $36 \mathrm{~m}$ are used to look at the observable variations induced by the perturbation noted "E" on Fig 2. The normalized difference - expressed as:

$$
\frac{\sum\left|\delta o b s_{S K}-\delta o b s_{P E}\right|}{\sum\left|\delta o b s_{P E}\right|} \times 100
$$

, where $\delta o b s_{S K}$ is the observable variation estimated with the T-A-SK, and $\delta o b s_{P E}$ the variation measured on PE simulations - is computed for the 550 rays for each size of the sub-array. The results in Table 1 show that the estimate error does not vary a lot with the array size. Results, presented in Table 1 show that the estimate error have a variation of less than $2 \%$ on the 3 observations, with respect to the array size. We estimate that the biggest difference being $1.4 \%$ between the smallest and the biggest sub-array, the sub-array size is not a crucial factor for the estimate quality.

Table 1: Normalized difference (Eq. 2) of the 550 rays for each observable and each sub-array size.

\begin{tabular}{ccccc}
\hline Sub-Array Size & $\mathbf{1 5} \mathbf{~ m}$ & $\mathbf{2 1} \mathbf{~ m}$ & $\mathbf{3 1} \mathbf{~ m}$ & $\mathbf{3 6} \mathbf{~ m}$ \\
\hline TT & $9.8 \%$ & $9.7 \%$ & $9.6 \%$ & $9.4 \%$ \\
DOA & $12.1 \%$ & $11.6 \%$ & $10.9 \%$ & $10.7 \%$ \\
DOD & $12.5 \%$ & $12 \%$ & $11.5 \%$ & $11.1 \%$ \\
\hline
\end{tabular}

However, the array size is determinant for the acoustic arrival separation. The bigger the sub-array is, the better is the raypath separation. In contrary, the bigger the sub-array is, the fewer sub-arrays 
can be made out of a single array. Therefore, a trade-off needs to be found to maximize the number of observables. In the follow up of the study, 21-m source-receiver arrays will be taken.

\subsection{The Sound-Speed Perturbation Location}

Four locations of a same sound-speed perturbation are represented on Fig. 2.

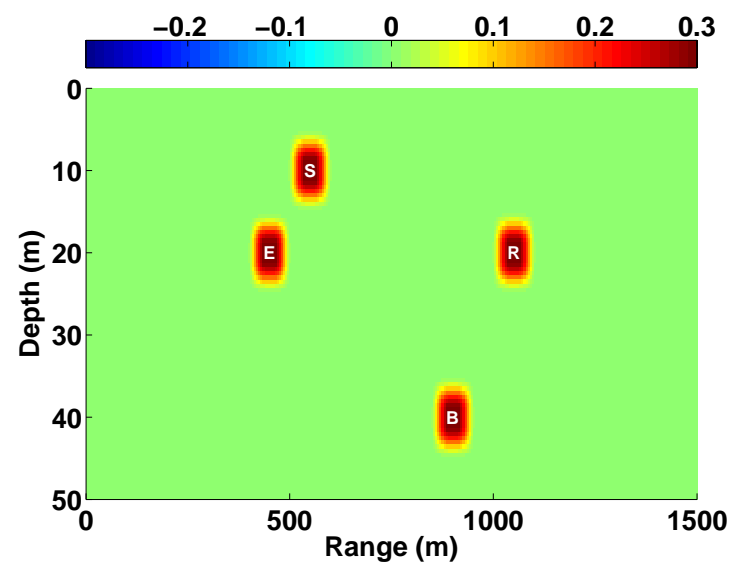

Figure 2: Pekeris waveguide with examples of three possible raypaths. The source and receive array at the beginning and the end of the waveguide have 97 elements evenly spaced by $0.5 \mathrm{~m}$ i.e. . These observables - measured with source-receiver arrays and the double beamforming technique [Roux et al. 2008 ] - vary when the sound speed distribution changes in the waveguide.

The normalized difference between the T-A-SK estimates and the PE measurement are summarized in Table 2 for each observable. It shows that the T-A-SK estimate is reliable no matter where the perturbations are located. Indeed, the T-A-SK-to-PE normalized difference differs, from one case to another, by only $0.5 \%$ at the maximum.

Table 2: Normalized difference (Eq. 2) of the 550 rays for each observable and each perturbation location shown on Fig. 2

\begin{tabular}{ccccc}
\hline Perturbation Location & Source (E) & Bottom (B) & Receiver (R) & Surface (S) \\
\hline TT & $9.8 \%$ & $9.7 \%$ & $9.6 \%$ & $9.9 \%$ \\
DOA & $11.6 \%$ & $11.7 \%$ & $11.4 \%$ & $11.9 \%$ \\
DOD & $11.8 \%$ & $12.2 \%$ & $11.9 \%$ & $12.3 \%$ \\
\hline
\end{tabular}

\subsection{The Sound-Speed Perturbation Value}

Several values of the sound-speed perturbation noted "E" on Fig. 2 are taken between $0.01 \mathrm{~m} / \mathrm{s}$ and 20 $\mathrm{m} / \mathrm{s}$ as shown on Fig. 3 .
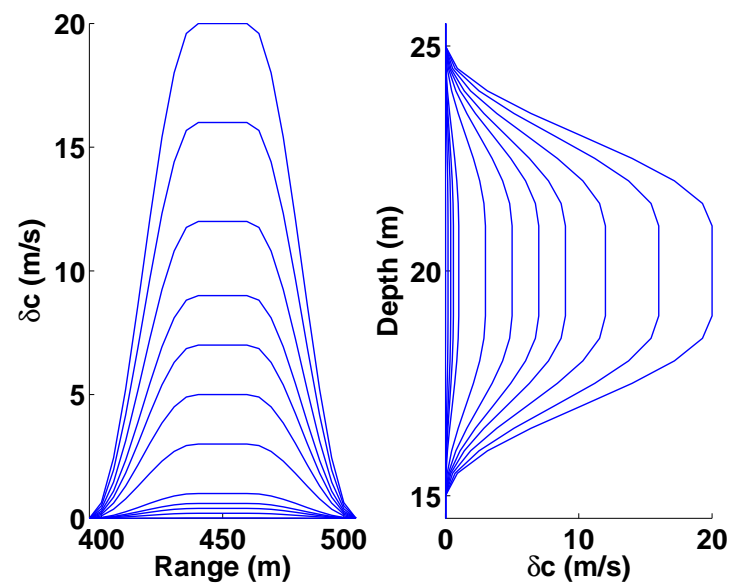

Figure 3: Shape of the sound-speed perturbations of different values between $0.01 \mathrm{~m} / \mathrm{s}$ and $20 \mathrm{~m} / \mathrm{s}$.

The goal here is to determine the validity domain of the first order Born approximation, which assumes relatively "small" sound-speed perturbations. As the first order Born approximation is used to linearize 
the forward problem in the T-A-SK theory, it is necessary to have a knowledge on the behavior of the observable variations with respect to the value of the sound speed perturbations. Results are exposed on Fig. 4. First, it can be noticed that the first acoustic arrivals (2 to 6 reflections) lose their linear behavior sooner that the last ones. For instance, rays with 2 reflections loose the linear behavior around $6 \mathrm{~m} / \mathrm{s}$ (Fig. 4a).

(a)

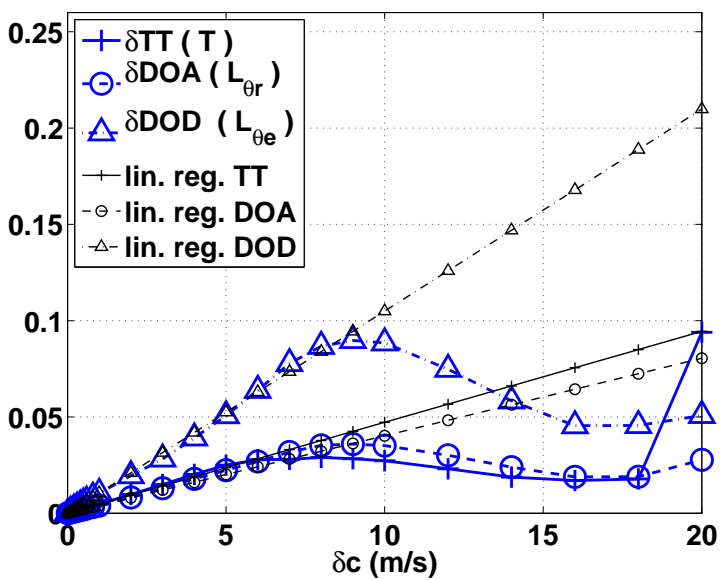

(b)

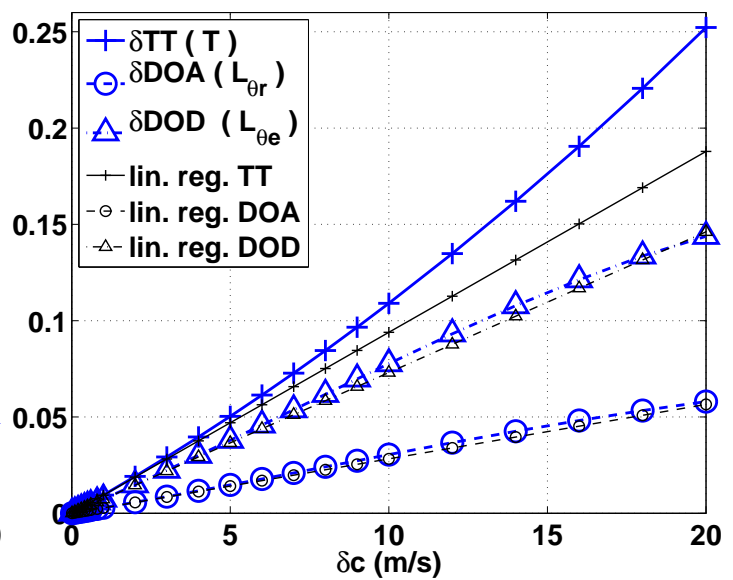

Figure 4: Fig. 4a shows the behavior of the observable variations of the rays with 2 reflections with respect to the sound-speed perturbation value. Fig. 4b shows the behavior of the observable variation of the rays with 7 to 12 reflections with respect to the sound-speed perturbation value.

Indeed 5, the wavefronts of the first acoustic arrivals become very irregular when the perturbation value increases and the acoustic arrivals become badly localized in the double-beamformed domain (see Fig. 5). Observable variations of the latest arrivals can be approximated linearly until sound-speed perturbations of $20 \mathrm{~m} / \mathrm{s}$ (see Fig. 4b for the observable variations of rays with 7 to 12 reflections).

(a)

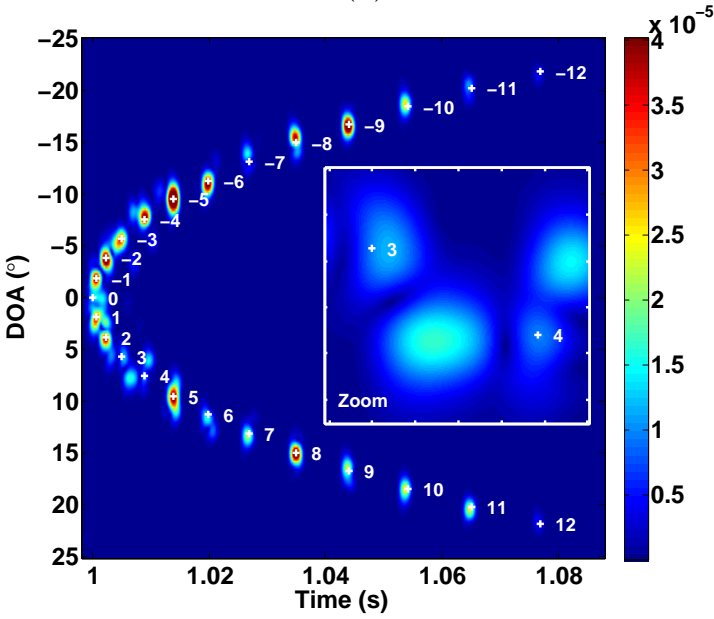

(b)

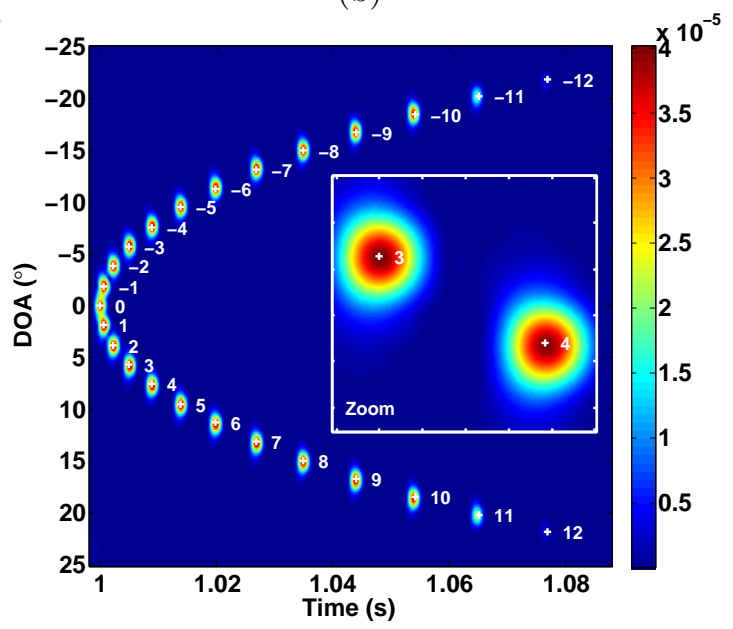

Figure 5: Double-beamformed 3D-signal (time, reception angle, emission angle) integrated over the emission-angle dimension with a zoom on the acoustic arrivals 3 and 4 . For the Fig. 5a the soundspeed perturbation has a value of $20 \mathrm{~m} / \mathrm{s}$ ( $1.3 \%$ of the sound-speed background) and for the Fig. $5 \mathrm{~b}, 0.1$ $\mathrm{m} / \mathrm{s}$ (6e-3 $\%$ of the celerity background).

\subsection{The Sound-Speed Perturbation Area}

The case of a large sound-speed perturbation (Fig. 6) is studied and compared to the "E"-case of Fig. 2.

The histogram of the absolute difference between the T-A-SK estimates of the observable variations and the corresponding PE measurements are compared in the case of a small and large perturbation and presented on Fig. 7.

Results show that the absolute difference between T-A-SK estimates and PE measurements increases a lot in the case of large perturbation. Indeed, the T-A-SK formulation of the forward problem supposes that the total effect of a sound-speed perturbation on the incident acoustic wave is the sum of the effects induced by elementary perturbation compounding the large perturbation. This assumption becomes 


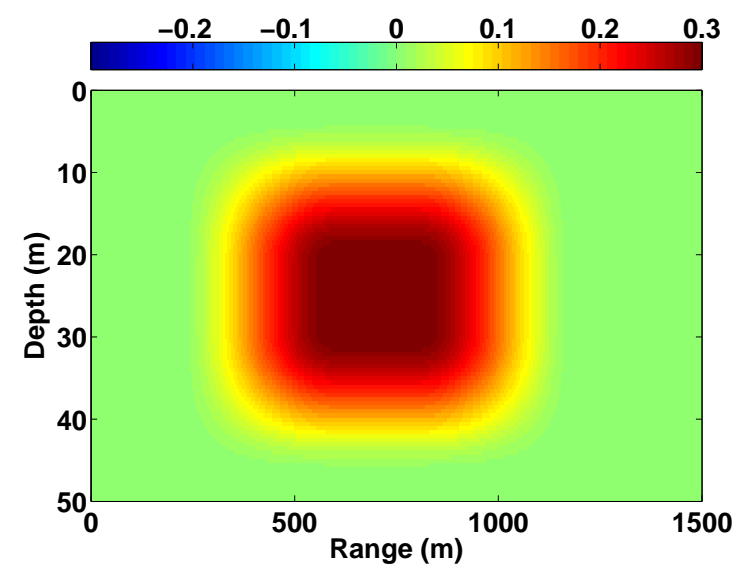

Figure 6: Map of the $0.3-\mathrm{m} / \mathrm{s}$ sound-speed perturbation of large area inside the waveguide.

(a)

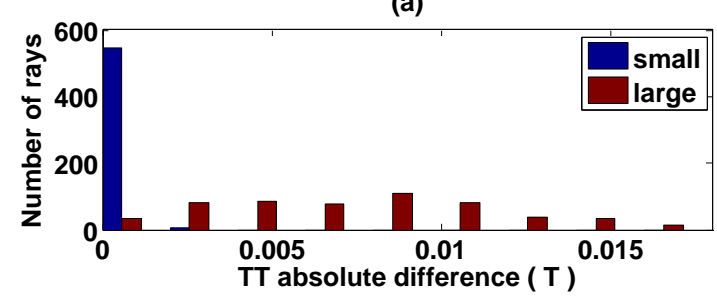

(b)

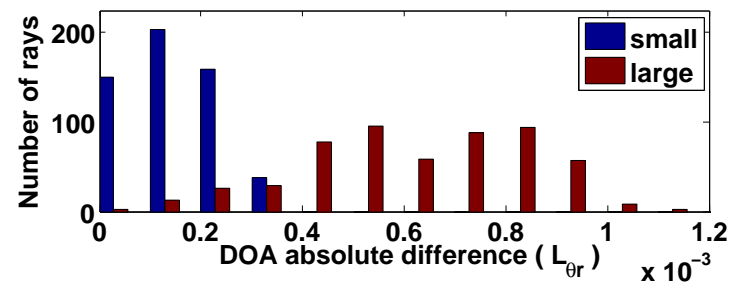

(c)

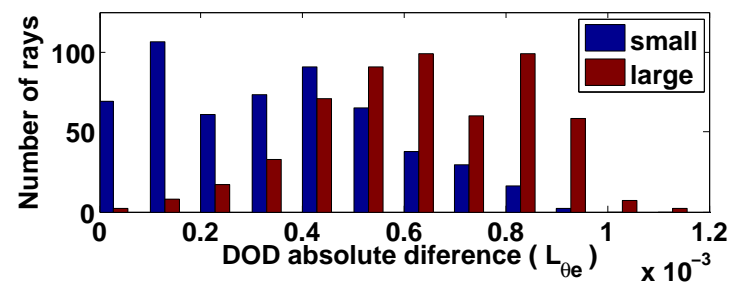

Figure 7: Histogram of the absolute difference between the T-A-SK estimates and the PE measurements of variations of each observable. $\mathrm{T}$ is the signal period at the central frequency $(0.4 \mu \mathrm{s}) . L_{\theta e}$ and $L_{\theta r}$ are the sizes of the main lobe of the 3D-signal in angle for the emission and the reception respectively $\left(\sim 3^{\circ}\right.$ for $21 \mathrm{~m}$ source-receiver sub-arrays). 
wrong when the perturbation is so large that the part of the perturbation close to the sources modifies dramatically the acoustic field that reaches the end of the perturbation.

\subsection{The Case of Multiple Sound-Speed Perturbations}

Finally, the case with multiple sound speed perturbations occurring simultaneously in the waveguide is studied (Fig. 8).

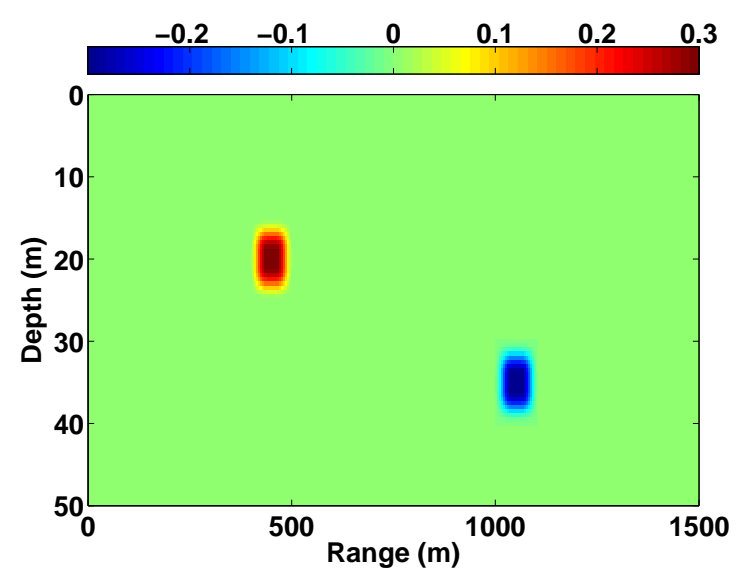

Figure 8: Map of the multiple sound-speed perturbations of opposite sign $(+/-0.3 \mathrm{~m} / \mathrm{s})$ inside the waveguide.

This case does not pose problem for two reasons:

1. Most of the acoustic paths pass by either one or the other perturbation but not both of them. Therefore, it can be considered as a single perturbation problem in this case.

2. For the acoustic paths that go through both the sound-speed perturbations, the effect of the first perturbation is sufficiently weak not to induce acoustic field fluctuations that cannot be approximated by the T-A-SK forward problem formulation.

\section{Conclusion}

The comparison between the T-A-SK formulation and the PE formulation of forward problem shows us the strength and the limits of the T-A-SK. Robust to array size, perturbation location and multiple perturbations, the T-A-SK linear approximation of the forward problem reaches its limits regarding the sound-speed perturbation values or areas. However in practice, sound-speed changes in the waveguide do not occur instantaneously. These limits can then be avoided if the time lapse between the reference acquisition and the perturbed acquisition is sufficiently small. This study also allows getting a priori information on the errors made on T-A-SK estimates. It might be useful to integrate this a priori information to improve the future inversion process.

\section{Acknowledgements}

The authors would like to acknowledge to Dr. J. Sarkar and Dr. B. Cornuelle from the Marine Physical Laboratory, SIO for their help on PE simulations and their useful discussions; and Dr. E. Skarsoulis from the Institute of Applied and Computational Mathematics, FORTH for his helpful comments.

\section{References}

[1] W. Munk and C. Wunsch, "Ocean acoustic tomography: a scheme for large scale monitoring", Deep Sea Research Part A. Oceanographic Research Papers 26, 123-161 (1979).

[2] M. Born and E. Wolf, Principles of optics: electromagnetic theory of propagation, interference and diffraction of light (Cambridge university press, Cambridge, UK) (1999).

[3] A. J. Devaney, "Geophysical Diffraction Tomography", IEEE Transactions on Geoscience and Remote Sensing GE-22, 3-13 (1984). 
[4] M. J. Woodward, "Wave-equation tomography", in SEG Annual Meeting, Anaheim, CA, 1232-1236 (Society of Exploration Geophysicists) (1988).

[5] H. Marquering, F. Dahlen, and G. Nolet, "Three-dimensional sensitivity kernels for finite-frequency traveltimes: the banana-doughnut paradox", Geophysical Journal International 137, 805-815 (1999).

[6] E. K. Skarsoulis and B. D. Cornuelle, "Travel-time sensitivity kernels in ocean acoustic tomography", Journal of the Acoustical Society of America 116, 228-238 (2004).

[7] P. Roux, B. D. Cornuelle, W. A. Kuperman, and W. S. Hodgkiss, "The structure of raylike arrivals in a shallow-water waveguide.", The Journal of the Acoustical Society of America 124, 3430-9 (2008).

[8] B. Nicolas, I. Iturbe, P. Roux, and J. Mars, "Double formation de voies pour la séparation et l'identification d'ondes: applications en contexte fortement bruité et à la campagne faf03", TS. Traitement du signal 25, 293-304 (2008).

[9] I. Iturbe, P. Roux, B. Nicolas, J. Virieux, and J. I. Mars, "Shallow-Water Acoustic Tomography Performed From a Double-Beamforming Algorithm: Simulation Results", IEEE Journal of Oceanic Engineering 34, 140-149 (2009).

[10] F. Aulanier, B. Nicolas, P. Roux, and J. I. Mars, "Direction-of-arrival, direction-of-departure and travel-time sensivity kernels obtained through double beamforming in shallow water", in Proceedings of the 4 th international conference and exhibition on Underwater Acoustic Measurements: Technologies and Results, volume 4, 453-460 (2011).

[11] F. Jensen, W. Kuperman, M. Porter, and H. Schmidt, Computational Ocean Acoustics (SpringerVerlag, New York, USA) (2011). 